\title{
Intrapleural delivery of mesenchymal stem cells: a novel potential treatment for pleural diseases
}

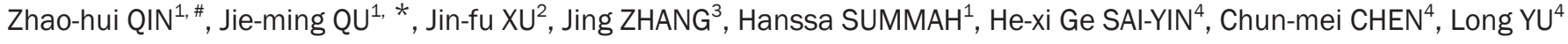 \\ ${ }^{1}$ Department of Pulmonary Medicine, Huadong Hospital, Shanghai Medical College, Fudan University, Shanghai 200040, China; \\ ${ }^{2}$ Department of Pulmonary Medicine, Shanghai Pulmonary Hospital, Tongji University, Shanghai 200433, China; ${ }^{3}$ Department of Pul- \\ monary Medicine, Zhongshan Hospital, Shanghai Medical College, Fudan University, Shanghai 200032, China; ${ }^{4}$ State Key Laboratory \\ of Genetic Engineering, Institute of Genetics, School of Life Sciences, Fudan University, Shanghai 200433, China
}

\begin{abstract}
Aim: To develop a method to deliver mesenchymal stem cells (MSCs) into the pleural cavity for the treatment of pleural diseases. Methods: MSCs were isolated from rat bone marrow of rats and labeled with 4',6-diamidino-2-phenylindole dihydrochloride (DAPI) or green fluorescent protein (GFP) using a lentiviral vector. Eighteen Sprague-Dawley (SD) rats were inoculated intrapleurally with $1 \times 10^{6}$ MSCs-DAPI. The distribution of the fluorescent cells was observed using fluorescent microscopy for the following $30 \mathrm{~d}$. Another 12 rats inoculated intrapleurally with $1 \times 10^{6}$ MSCs-GFP were observed for $14 \mathrm{~d}$.

Results: The isolated cells were typical MSC phenotypes and could differentiate into adipocytes, osteoblasts, and chondroblasts in vitro. Microscopic analysis revealed that the labeled cells adhered to the surface of the pleural cavity. The highest number of the labeled cells was found to be adhered to all specimens from the mediastinal pleura, but no labeled cells were detected in the lung parenchyma or other tissues/organs, such as the liver, kidney, spleen, and mesenterium. Incidentally, stomas were found in the mediastinal pleura. The recovered MSCs-GFP from the pleural cavity retained their ability to adhere and proliferate.

Conclusion: We have established a novel method for intrapleural delivery of MSCs. The distribution of intrapleurally delivered MSCs was found to be limited to the pleurae and the pleural cavity, thereby providing us with a new approach to further investigation of the therapeutic roles of MSCs in pleural diseases.
\end{abstract}

Keywords: mesenchymal stem cells (MSCs); stem cells transplantation; pleural cavity; pleural diseases; lung

Acta Pharmacologica Sinica (2011) 32: 581-590; doi: 10.1038/aps.2011.22; published online 2 May 2011

\section{Introduction}

Primary and secondary pleural diseases such as malignant pleural mesothelioma, pleural metastasis of lung cancer, pneumothorax, pleurisy and pleural effusions are common chest diseases involving the pleura. Despite advancement in different diagnostic tools and in the array of treatment options available today, effective treatment of pleural diseases such as malignant pleural mesothelioma, malignant pleural effusion and recurrent pneumothorax still remains challenging ${ }^{[1-4]}$.

Recent studies, including one of our previous studies, have demonstrated that intravenous (iv) or intratracheal (it) delivery of bone marrow-derived mesenchymal stem cells (MSCs) participates in the improvement of various experimental

\footnotetext{
\# Now in Department of Pulmonary Medicine, Longhua Hospital, Shanghai University of Traditional Chinese Medicine, Shanghai 200032, China.

* To whom correspondence should be addressed.

E-mail jmqu64@yahoo.com.cn

Received 2010-08-27 Accepted 2011-03-10
}

models of lung injury, thus demonstrating that MSCs play an important role in the treatment of animals with acute lung injury ${ }^{[5-12]}$.

Because the pleural cavity is a tightly closed compartment, we hypothesized whether intrapleurally delivered MSCs could survive and adhere to the host pleura, and whether these cells could help to modulate the repair process of the injured pleura, thus intrapleural MSCs could become a new cell-based therapeutic strategy for pleural diseases. In the current study, we observed the dynamic distribution of intrapleurally delivered MSCs with the aim of validating this new route for the delivery of MSCs as a potential treatment method for pleural diseases.

\section{Materials and methods}

Animal care

The animals used in this study were specific-pathogen-free male Sprague-Dawley rats (6-8 weeks old; Slac Laboratory 
Animal Co Ltd, Shanghai, China). The rats received humane care in compliance with the NIH principles of laboratory animal care and the Chinese national regulations for experimental animal care. All protocols were approved by the Animal Care and Use Committee of Fudan University.

\section{Isolation, purification, and expansion of MSCs}

MSCs were isolated from rat bone marrow as described in previous studies ${ }^{[8]}$. In brief, whole marrow was flushed from the tibias and femurs of Sprague-Dawley rats (4-weeks old, male) with ice-cold Dulbecco's modified Eagle's medium/F12 (DMEM/F12; Gibco, Carlsbad, CA, USA). The cells were washed and plated in plastic flasks at $2 \times 10^{6}$ cells $/ \mathrm{mL}$ in DMEM/F12 containing 10\% fetal bovine serum (FBS), 100 units/mL penicillin, and $100 \mu \mathrm{g} / \mathrm{mL}$ streptomycin (Gibco, Carlsbad, CA, USA). The cell suspension was incubated at $37{ }^{\circ} \mathrm{C}$ with $5 \% \mathrm{CO}_{2}$; nonadherent cells were discarded $48 \mathrm{~h}$ later. For routine maintenance, the medium was replaced twice weekly and cells were subcultured at a one-to-three split ratio by trypsinization $(0.25 \%$ trypsin/ $1 \mathrm{mmol} / \mathrm{L}$ EDTA; Gibco, Carlsbad, CA, USA). Cells were further purified by passages.

\section{FACS analysis of MSCs}

MSCs were analyzed by FACS using antibodies for cell surface markers CD11b, CD45, CD29, CD73, and CD90, as described previously ${ }^{[13]}$. Briefly, MSCs from passage 3 were trypsinized and counted after rinsing in PBS ( $\mathrm{pH} 7.2$ ). Aliquots of $5 \times 10^{5}$ cells were incubated for $30 \mathrm{~min}$ with PE/FITC labeled monoclonal antibodies against rat CD11b, rat CD45, rat CD29, rat CD90 (BioLegend, San Diego, CA, USA), purified mouse antiCD73 (BD Pharmingen ${ }^{\mathrm{TM}}$, San Diego, CA, USA), donkey antimouse IgG-FITC (Santa Cruz Biotechnology, Santa Cruz, CA, USA), and isotype-matched FITC- and PE-conjugated negative controls (IgG1-FITC/IgG1-PE, IQ products, CA, USA) according to the manufacturer's recommendations. After being washed with PBS, the stained cells were analyzed on a flow cytometer (Beckman Coulter Epics XL, USA) to identify the phenotypes of MSCs.

\section{Differentiation assays}

Passage 2 MSCs cultured with DMEM/F12 containing 10\% FBS were used for the differentiation assay ${ }^{[6]}$. Adipogenic differentiation was induced by seeding the MSCs for $21 \mathrm{~d}$ in DMEM/F12 containing 10\% FBS supplemented with 1 $\mu \mathrm{mol} / \mathrm{L}$ dexamethasone (Sigma-Aldrich, USA), $10 \mu \mathrm{g} / \mathrm{mL}$ insulin (Sigma-Aldrich, USA), $200 \mu \mathrm{mol} / \mathrm{L}$ indomethacin, and $0.5 \mathrm{mmol} / \mathrm{L}$ IBMX. The cells containing droplets of fat were identified by staining the culture with 3.75\% Oil Red. Osteogenic differentiation was induced by cultivating the MSCs in DMEM/F12 containing 10\% FBS and supplemented with $10 \mathrm{mmol} / \mathrm{L} \beta$-glycerol phosphate, $50 \mu \mathrm{mol} / \mathrm{L}$ ascorbic acid, and $0.1 \mu \mathrm{mol} / \mathrm{L}$ dexamethasone for 14 to $21 \mathrm{~d}$. Alkaline phosphatase (AKP) levels were investigated by staining cells using AKP kits at d 14. The calcium-containing precipitates were visualized after staining with $2 \%$ Alizarin Red S
(Sigma-Aldrich, USA) adjusted to a $\mathrm{pH}$ of 4.2 with ammonium hydroxide at $\mathrm{d} 21$. Chondroblast differentiation was induced by cultivation of the MSC in DMEM/F12 containing $0.25 \%$ FBS supplemented with $0.1 \mu \mathrm{mol} / \mathrm{L}$ dexamethasone, 50 $\mu \mathrm{g} / \mathrm{mL}$ ascorbic acid, $10 \mathrm{ng} / \mathrm{mL}$ transforming growth factor beta 1 (TGF- $\beta 1$ ), and 1\% ITS (Sigma-Aldrich, USA). Toluidine blue staining was used to demonstrate chondroblast differentiation.

\section{DAPI labeling of MSCs and growth characteristics assays}

MSCs were labeled with 4',6-diamidino-2-phenylindole dihydrochloride (DAPI; cell culture tested by Sigma, St Louis, $\mathrm{MO}, \mathrm{USA}$ ) in vitro prior to in vivo inoculation into the pleural cavity. For labeling, MSCs were incubated for $4 \mathrm{~h}$ in DMEM/ F12 medium containing $50 \mu \mathrm{g} / \mathrm{mL}$ DAPI. Cells were washed 6 times with PBS and then dissociated into a single cell suspension with $0.25 \%$ trypsin $/ 1 \mathrm{mmol} / \mathrm{L}$ EDTA for further analysis $^{[8]}$.

Growth curves were generated to ascertain whether DAPI labeling would affect the growth characteristics of MSCs. Passage 2 MSCs labeled or not labeled with DAPI were typsinized, counted, seeded in 6-well plates, and cultured with DMEM/F12 containing 10\% FBS. MSCs or MSCs-DAPI were seeded at the same cell density $\left(3 \times 10^{4} /\right.$ well $)$ in a total of 12 cell culture plates. For the next $12 \mathrm{~d}$, the cells were counted using a microscope and a hemocytometer on a daily basis. The average cell number of three wells for each time point was calculated to plot the cell growth curve.

\section{Green fluorescent protein (GFP) labeling of MSCs}

Because GFP is expressed by the labeled cell itself, its fluorescent signal does not attenuate with cell division, and its fluorescent signal is more stable than that of DAPI. To compare with and validate the experimental results of the DAPI labeling method, GFP labeling of MSCs was also used in this study. MSCs $\left(1 \times 10^{5}\right)$ were incubated for $8 \mathrm{~h}$ with pGC FU-GFPLentivirus (Shanghai GeneChem Co, Ltd, China) at a multiplicity of infection (MOI) of 10 and $5 \mu \mathrm{g} / \mathrm{mL}$ of polybrene. The supernatant was then discarded, and the transfected cells were cultured and passaged in DMEM/F12 medium containing 10\% FBS by trypsinization followed by splitting at a 1:3 ratio. The presence of MSCs-GFP cells and the intensity of GFP fluorescence were observed by fluorescent microscopy. MSCs labeled with GFP were dissociated into a single cell suspension using $0.25 \%$ trypsin/ $1 \mathrm{mmol} / \mathrm{L}$ EDTA for further intrapleural administration.

\section{Inoculation of MSCs-DAPI or MSCs-GFP into the pleural cavity}

Six-to-eight week-old rats were anesthetized using diethyl ether inhalation. A 5-mm incision was made on the right chest through the skin and the subcutaneous soft tissues. MSCsDAPI $\left(1 \times 10^{6}\right.$ cells in $750 \mu \mathrm{L}$ DMEM/F12) were injected into the pleural cavity using a $20 \mu \mathrm{L}$ plastic pipette tip, instead of a needle, connected to a $1 \mathrm{~mL}$ syringe so as to avoid possible pneumothorax or lung injury that could have been caused by a needle. After cell transplantation, the skin incision was closed 
with sutures.

A total of 20 naive rats were inoculated into the right pleural cavity with MSCs-DAPI; fluorescent cells were traced in 16 rats at four different time points over a period of $30 \mathrm{~d}(n=4 ; \mathrm{d} 1,4$, 14 , and 30 ). The remaining 4 rats ( 1 rat for each time point) were used to recover the viable MSCs-DAPI in vivo from their pleural cavity to see whether the normal features of these cells had been changed when cultured in vitro again. Fifteen rats were used for MSC-GFP inoculation, 12 of which were used to trace the fluorescent cells over a period of $14 \mathrm{~d}(n=3 ; \mathrm{d} 1,4,7$, and 14), and 3 rats were used to recover the viable MSCs-GFP in vivo from the pleural cavity at $\mathrm{d} 1$ only. The $\mathrm{d} 7$ time point was added and d 30 was omitted to better trace the fluorescent cells in the early days.

\section{Sample collection}

Rats were anesthetized using diethyl ether inhalation and the peritoneal cavity was opened. Animals were then sacrificed by incision of the abdominal aorta. After collection of the liver, kidney, spleen and mesenterium samples, both the right and the left pleural cavities were fixed for $15 \mathrm{~min}$ by injection of $2 \mathrm{~mL} \mathrm{4 \%}$ paraformaldehyde into each side through the diaphragm. Specimens from both the right and the left thorax were collected for further analysis, including pleural fixative fluid; whole lungs; mediastinal pleura, including the pleura on the left and right sides of the right lung accessory lobe; pericardium located at the left and right sides of heart; whole diaphragms; parts of chest walls; the thymuses; and the heart.

It is necessary to point out that, different from humans, rats have an additional lobe in the right lung, the right accessory lobe. Located either at its left or right side is a free membrane that belongs to the mediastinal pleura. The term "visceral pleura" only refers to the pleura on the surface of the lung, while "parietal pleura" refers to the pleura on the surface of the pleural cavity, except the visceral pleura. The mediastinal pleura, including the pericardium, the pleurae on the surfaces of the diaphragms, and the inner side of chest walls and thymuses, are all parietal pleurae. However, the epicardium, which is located on the surface of heart, does not belong to either the visceral or the parietal pleura.

\section{Histological analysis}

The mediastinal pleura, mesenterium and translucent diaphragms collected from the animals were mounted on glass slides and visualized under a florescence microscope. Paraformaldehyde-fixed pleural cavity fluid and translucent thymus were also examined directly under a fluorescence microscope. Other solid organs or tissues, including the lungs, heart, liver, kidney, spleen, and chest wall, could not be observed directly under a microscope, so the surfaces of these organs and the inner sides of the chest wall were carefully sliced into very thin sections with a sharp razor blade. With the upper sides of the sections attached closely to the surfaces of slides, the presence of fluorescence was visualized under a fluorescence microscope.

\section{Harvest of cells from the pleural cavity}

Into each pleural cavity $0.25 \%$ trypsin $/ 1 \mathrm{mmol} / \mathrm{L}$ EDTA (3 $\mathrm{mL}$ ) was injected through the diaphragm. After $6 \mathrm{~min}$, digested cells were collected with a needle connected to a 10 $\mathrm{mL}$ syringe inserted into the cavity through the lower part of the diaphragm. Using this method, more than $90 \%$ of the digestive solution was recovered. Then trypsinization was terminated with DMEM/F12 medium containing 10\% FBS, and the recovered cell suspension was centrifuged. Cells were resuspended and cultured in DMEM/F12 containing 10\% FBS for further analysis.

\section{Exchange of fluids and cells between the left and right pleural cavities}

Any diffusion of pleural fluid and injected MSCs between the left and right pleural cavity was determined by injection of methylene blue dye and DAPI-labeled MSCs, respectively. Briefly, five animals were anesthetized by diethyl ether inhalation and exsanguinated by incision of the abdominal aorta. A small opening was made on the right chest wall, and the left pleural cavity was carefully exposed, without damaging the mediastinal pleura. Methylene blue dye $(3 \mathrm{~mL}, 10 \mathrm{mg} / \mathrm{mL})$ or DAPI-labeled MSCs $\left(1 \times 10^{6}\right.$ cells in $3 \mathrm{~mL}$ PBS) were then injected into the right pleural cavity through the small opening. At the same time, we observed whether the injected fluids and cells penetrated through the mediastinal pleura to the left side.

\section{Microscopic observation for channels between the left and right pleural cavities}

Both normal and DAPI-stained mediastinal pleurae were visualized under light and fluorescence microscopes to investigate the presence of channels that could be responsible for the exchange of fluid and cells. Rats were anesthetized by diethyl ether inhalation, and mediastinal pleurae were stained for $4 \mathrm{~h}$ by injecting $2 \mathrm{~mL}$ DMEM/F12 medium containing $50 \mu \mathrm{g} / \mathrm{mL}$ DAPI into the right pleural cavity. The rats were then reanesthetized and the pleural cavities were fixed with $4 \%$ paraformaldehyde for $15 \mathrm{~min}$. The rats were finally sacrificed, and the DAPI-stained mediastinal pleurae were collected for further microscopic observation. In addition, normal mediastinal pleurae were also collected in a similar way and observed under a light microscope.

\section{Statistical analysis}

Statistical analysis was done using chi-square test. A $P$ value of $<0.05$ was considered statistically significant.

\section{Results}

\section{Identification of MSC phenotypes}

Various cell-specific markers of MSCs were analyzed by FACS to determine the cell phenotypes present in our experiments. Consistent with previous studies ${ }^{[13,14]}$, our results revealed that the majority of MSCs were CD29, CD73, and CD90 positive. However, only a small proportion of isolated cells were 
positive for hemopoietic cell surface makers CD11b/c and CD45 (Figure 1). The results of isotype-matched FITC- and PE-conjugated negative controls are shown in Figure S1. A differentiation assay demonstrated that the cells could differentiate into adipocytes, osteoblasts, and chondroblasts under standard in vitro differentiating conditions, which means that the cells have multipotent differentiation potential (Figure 2).
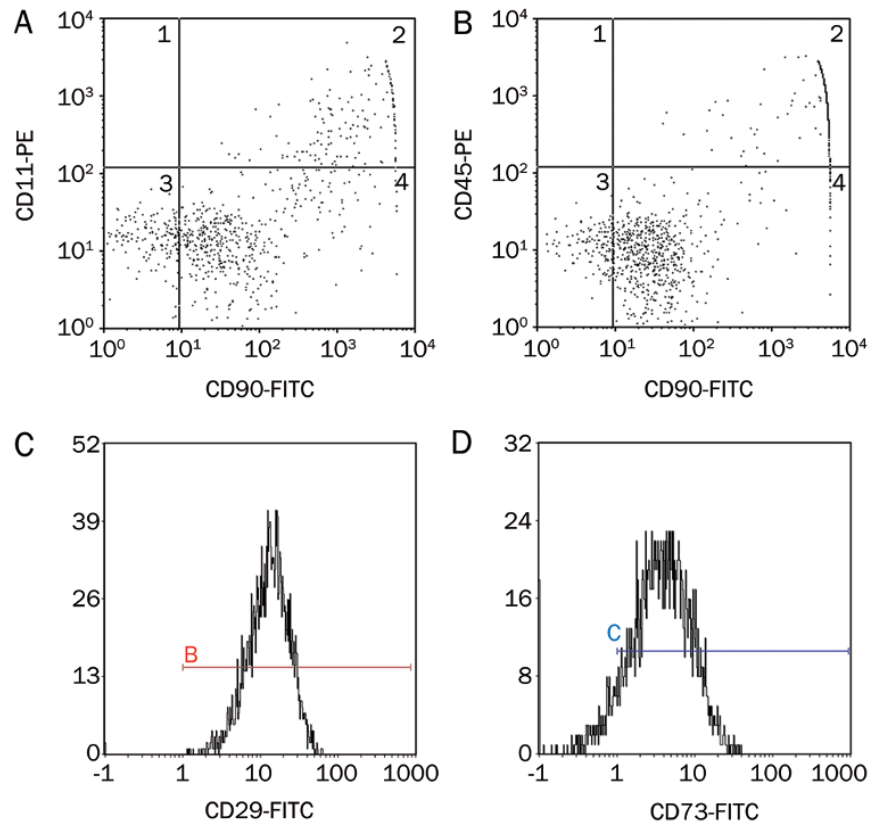

Figure 1. Characterization of MSC phenotype. Flow cytometry of MSCs demonstrated that majority of MSCs were CD90 (A, 95.84\%; B, 98.31\%), CD29 (C, 99.7\%) and CD73 (D, 89.5\%) positive. In contrast, only a small portion of isolated cells were positive for hemopoietic cell surface makers, CD11b/c (A, 1.86\%) and CD45 (B, 1.59\%).

Influence of DAPI labeling on growth of MSCs and stability of GFP expression

After incubation in DAPI $(50 \mu \mathrm{g} / \mathrm{mL})$ for $4 \mathrm{~h}$, an intensive fluorescent signal was found in the nuclei of the MSCs (Figure 3A-3C). In addition, strong fluorescent signal was present in the labeled cells even after $10 \mathrm{~d}$ (Figure 3D-3F), indicating that DAPI can be used to label MSCs effectively for at least 10 d. Growth curves of unlabeled MSCs vs DAPI-labeled MSCs showed that the DAPI-labeling method decreased the proliferation rate of MSCs by a longer lag phase and longer population doubling time (Figure S2). This result confirmed that DAPI was toxic to MSCs. After transfection with pGC FUGFP-Lentivirus, MSCs were found to be expressing GFP even after $29 \mathrm{~d}$ (Figure 3I). The efficiency of MSCs-GFP transfection was nearly $100 \%$ (Figure 3G-3I).

\section{Distribution of MSCs in the plurae of rats}

Microscopic analysis revealed the presence of DAPI-labeled MSCs in various tissues, including the visceral pleura and the
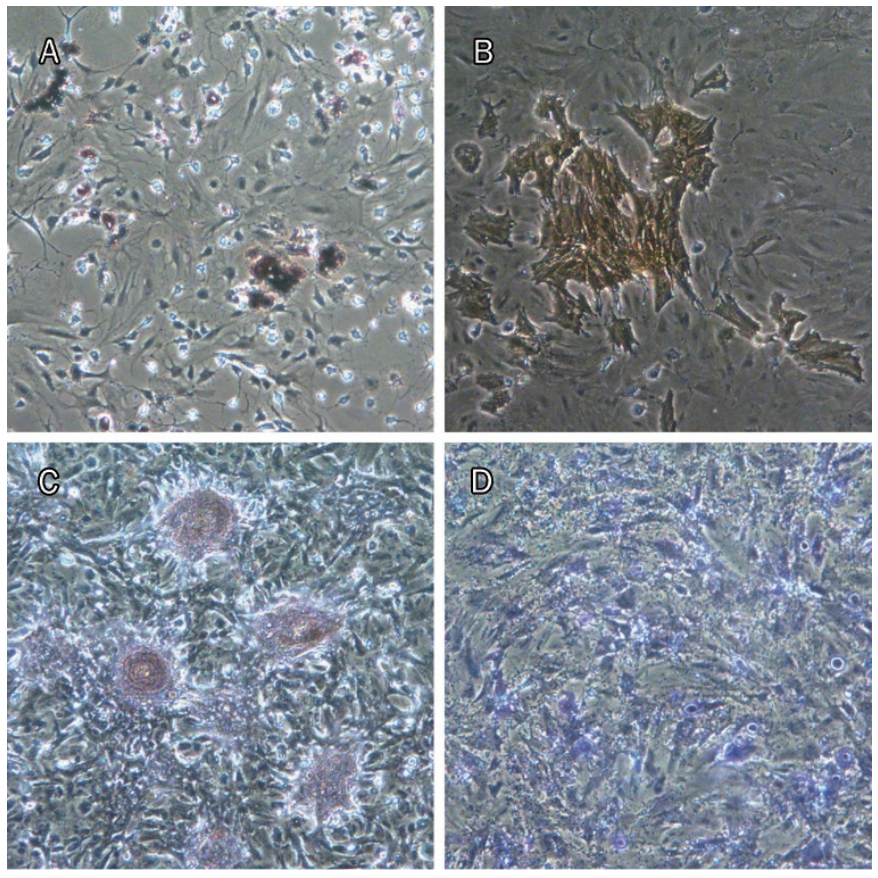

Figure 2. MSCs demonstrated multipotent property under standard in vitro differentiating condition. Differentiation into adipocytes (Oil Red staining, red) (A), osteoblasts (AKP staining, orange) (B) and (Alizarin staining, red) (C), and chondroblasts (Toluidin blue staining, blue) (D) was seen.

parietal pleura, as well as on the surface of epicardium (Figure $4,5)$. While DAPI-labeled cells were found in all 64 mediastinal pleura specimens, including the cardiac pericardium (100\%), and in all 32 pleural fixative solutions (100\%), they were found less frequently in tissues such as the epicardium, the parietal pleura of both the left and the right chest wall, and both the left and the right diaphragm (Figure 4). Here, the mediastinal pleura specimens included the pleura located in both the left and the right sides of the right lung accessory lobe and the pericardium located in both the left and the right sides of the heart. Therefore, 4 mediastinal pleura specimens were collected for each rat. A total of 16 rats were used in the DAPI-labeling method, so the total specimen number was 64 . No labeled cells were detected in the other tissues/organs, including the liver, kidney, spleen and the mesenterium (only the kidney is shown in Figure 5L). The labeled cells formed aggregates on the tissue surface and could be removed by scraping with a needle under a fluorescence microscope. Therefore, it seemed that inoculated MSCs adhered only to surfaces of these tissues. Overall, the number of adhered MSCs appeared to decrease with time because they could be less easily visualized in the same area of the microscopic field. A large number of labeled MSCs was also found in all 32 pleural fixative fluid specimens retrieved from each side of the pleural cavities, indicating that a significant portion of injected MSCs were in suspension rather than adhered to different surfaces during the observed period (Figure 5K). Interestingly, MSCs inoculated into the right pleural cavity were found to 

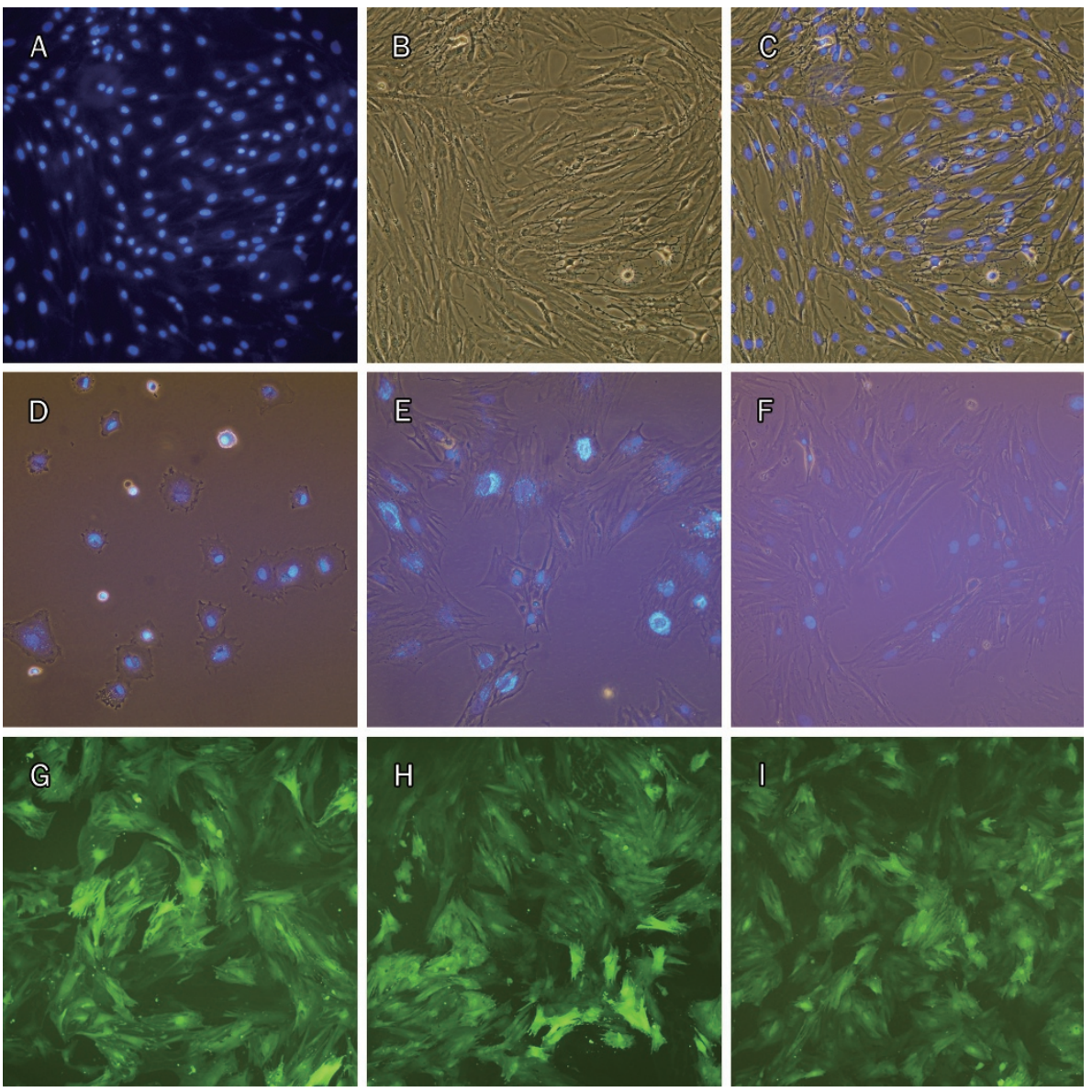

Figure 3. MSCs labeled with DAPI and GFP. MSCs stained with DAPI for $4 \mathrm{~h}$, fluorescence microscopy (A), phase contrast (B) and merge (C). MSCs nuclei were stained and the fluorescent signal was high. MSCs harvested from above cells were cultured in vitro for $4 \mathrm{~h}$ (D, merge), $4 \mathrm{~d}$ (E, merge), and $10 \mathrm{~d}(\mathrm{~F}$, merge, $2 \mathrm{~d}$ after passaged once). Relatively strong fluorescent signal was present in the cells even after $10 \mathrm{~d}$ post-labeling. Fluorescence microscopy of MSCs after transfection with pGC FU-GFP-Lentivirus for $7 \mathrm{~d}(\mathrm{G}), 19 \mathrm{~d}(\mathrm{H}, 9$ $\mathrm{d}$ after passaged once), and $29 \mathrm{~d}$ (I, $2 \mathrm{~d}$ after passaged twice). (A-F), $\times 200 ;(G-I), \times 100$.

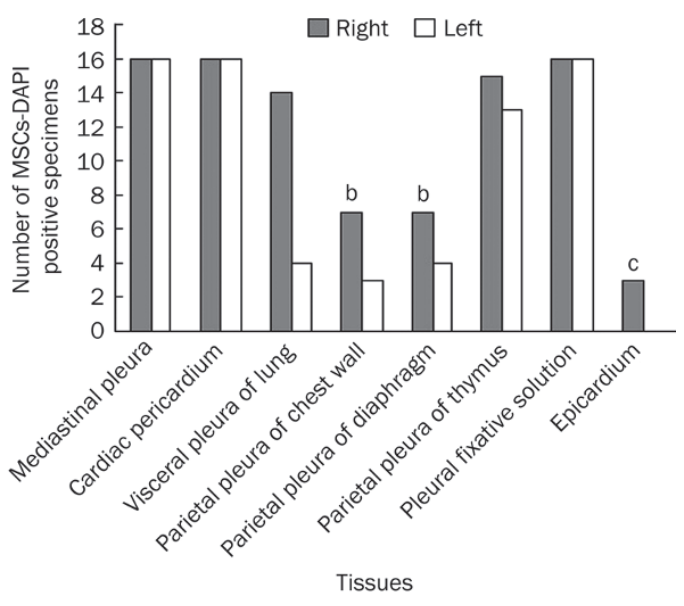

be present in the left pleural cavity (Figure 4,5), although the number of positive samples was less when compared to the right side (Figure 4). Figure 5 shows that MSCs adhered in the form of either cell aggregates or scattered cells. In the early phase $(\mathrm{d} 1,4)$, cell aggregates were more common than scattered cells. MSCs also showed a trend to adhere near small blood vessels (Figure 5A, 5C, 5D, Figure 6L).

It was also noted that the DAPI fluorescent signal of MSCs, although becoming weaker, was still strong enough to be
Figure 4. The MSCs-DAPI positive number of different tissues within two pleural cavities and pleural fixative solutions collected from total 16 experimental rats. For each bar, the maximum number was 16 , since the tissue it represents was collected from 16 rats. While DAPI-labeled cells were found in all 64 mediastinal pleura specimens including the cardiac pericardium (100\%) and in all 32 pleural fixative solutions (100\%), they were found less frequently in other tissues like the epicardium $(3 / 16=19 \%)$, the parietal pleura of both left and right chest wall $(10 / 32=31 \%)$ and both left and right diaphragm $(11 / 32=34 \%)$. In this figure, the column of mediastinal pleura represents only pleura located at both sides of right lung accessory lobe, while pericardium, which also belongs to the mediastinal pleura, was shown in a separate column. Right or Left, specimens collected from the right or left side of pleural cavities. ${ }^{\mathrm{b}} P<0.05,{ }^{\mathrm{c}} P<0.01$, compared with the mediastinal pleura including the pericardium.

detected even $30 \mathrm{~d}$ after MSC inoculation into the pleural cavity (Figure 5I-5K), implying that the fluorescent signal of DAPI in MSCs can be traced in vivo for a long time.

Tracking of MSCs-GFP showed similar results as DAPI labeling. MSCs-GFP were easily found in mediastinal pleura specimens at all four time points and in the translucent thymus at $\mathrm{d} 1$ (Figure 6). As GFP is expressed in the cytoplasm, the shape of the cells is clearly shown (Figure 6K). "Halos" around MSCs-GFP and MSCs-DAPI aggregates on the medi- 

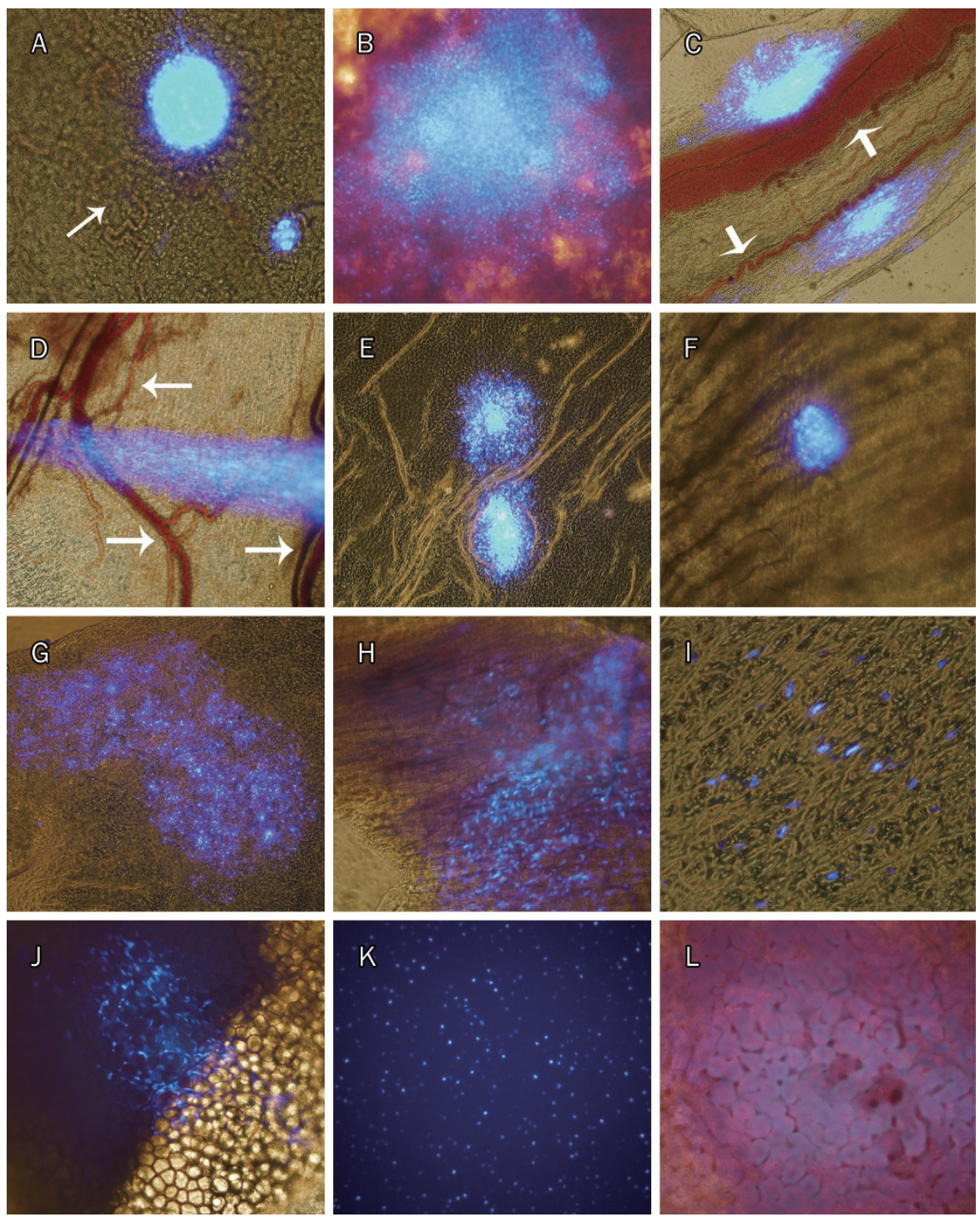

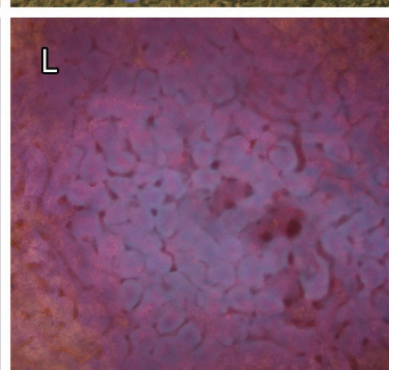

Figure 5. Destination of MSCs-DAPI after being inoculated into right pleural cavity in healthy rats. All were merged images. Images from d 1: the right cardiac pericardium $(A)$ and the surface of right lung (B). Images from d 4: the mediastinal pleura located at the right side of the right lung accessory lobe (C), the membranous part of right diaphragm (D), the left cardiac pericardium $(E)$, and inner surface of the right chest wall $(F)$. Images from d 14: the mediastinal pleura located at the right side of the right lung accessory lobe $(G)$, and the surface of epicardium $(H)$. Images from d 30: the mediastinal pleura located at the left side of the right lung accessory lobe (I), the surface of the left thymus $(J)$, the pleural fixative solution from the left thorax (K), and the surface of kidney (L). Of all microphotographs, $(B-E)$ and $(G), \times 100 ;(A),(F),(H)$, and $(J-L)$, $\times 200$; and $(I), \times 400$. This figure showed that the cells can adhere only to the surfaces of two pleural cavities (ie left and right), including on the surface of the epicardium. MSCs adhered in the form of either cell mass of different shape or scattered cells, and found to be proned to adhere near small blood vessels (white arrows in A, C, D). No DAPI labeled cell was found on the surface of kidney. astinal pleurae were often visualized (Figure 6C, Figure S3) on $\mathrm{d} 1$ but not at any of the following time points. MSCs-GFP were rarely found in pleural fixative fluids. Furthermore, no MSCs-GFP were found in the remaining specimens, including surfaces of the lungs, the heart, the chest wall, and the diaphragm.

\section{Channels exist for MSCs to traverse between the right and left pleural cavities}

An unexpected finding from our study was the migration of MSCs from the right pleural cavity to the left one and adherence to the surface in the left pleural cavity (Figure 4,5). This observation suggests that a channel exists in the mediastinal pleura that allows MSCs to penetrate to the other side. To further confirm this possibility, we injected methylene blue dye or a DAPI-labeled MSC suspension into the right pleural cavity and observed whether the dye or labeled cells could be found in the left pleural cavity. We found that the methylene blue dye was present in the non-injected left cavity just a few seconds after dye injection into the right pleural cavity (Figure 7A). Importantly, labeled MSCs inoculated into the right pleural cavity quickly migrated into the left pleural cavity along with the fluid (Figure 7B). Further microscopic observation of normal and DAPI-stained mediastinal pleura revealed that there were many oval stomas of different sizes in the cell monolayer of the mediastinal pleura (Figure 7C-7D), indicating that these stomas may be the channels mentioned above.

\section{Growth characteristics of inoculated MSCs recovered from the pleural cavity}

Mixed with many non-fluorescent native cells from the pleural cavity, a large number of viable MSCs-DAPI were visualized by fluorescence microscopy in the digestive solution recovered from the pleural cavity, even $30 \mathrm{~d}$ after inoculation (Figure $8 \mathrm{D}-8 \mathrm{~F})$. When cultured in vitro again, the recovered MSCsDAPI from all four time points still exhibited a capacity to adhere to plastic (Figure 8A-8F; only d 14 and d 30 are shown here). However, especially when passaged, the recovered 

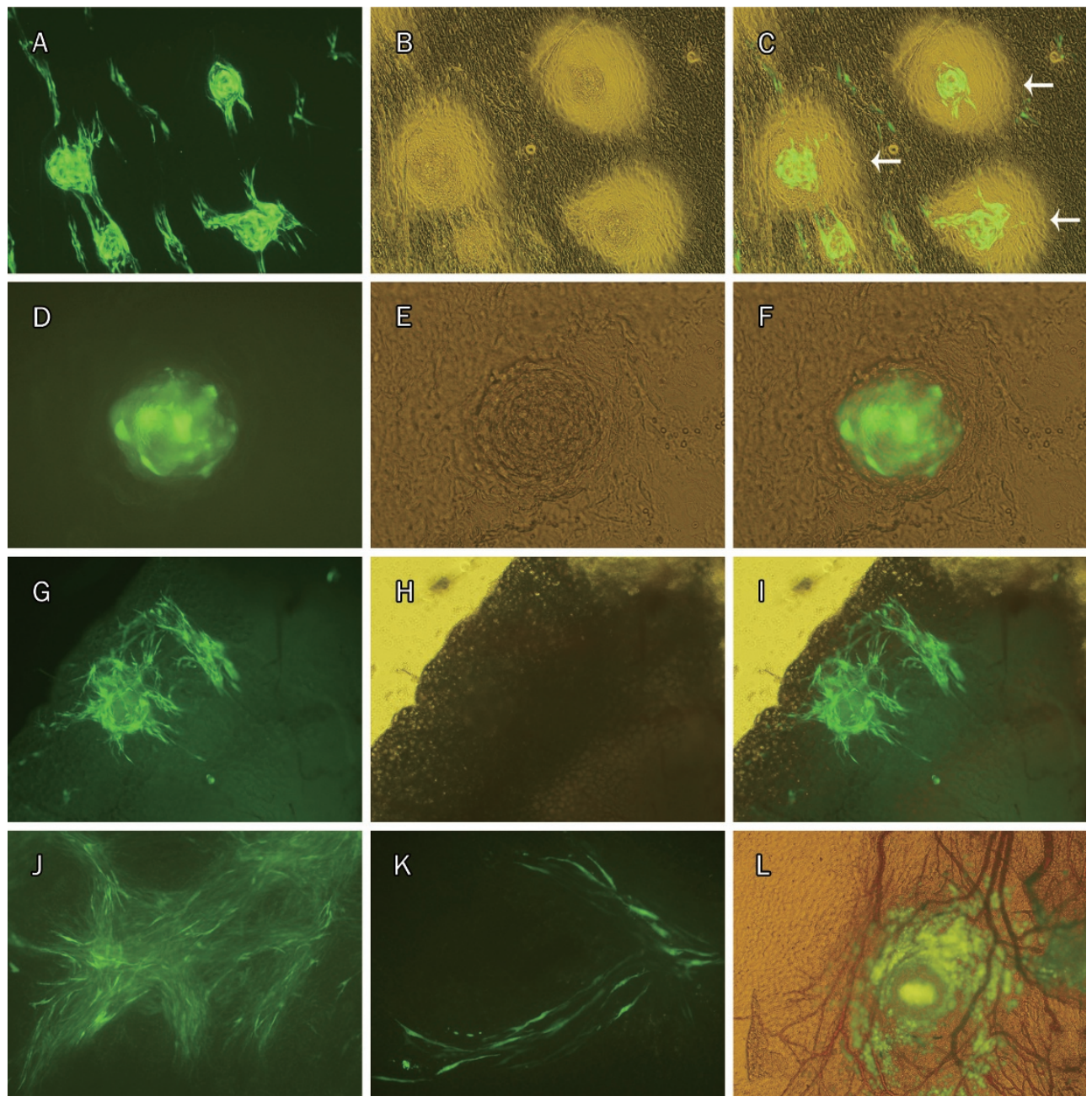

Figure 6. Destination of MSCs-GFP after being inoculated into right pleural cavity in healthy rats. Images from d 1 : the mediastinal pleura $(A-F)$, the right thymus $(G-1)$; d 4 : the mediastinal pleura $(J)$; $d$ : the mediastinal pleura (K); d 14: the mediastinal pleura (L). "Halo" around MSCs-GFP aggregate was seen in image $C$ (white arrows), indicating that interactions between MSCs and the pleural constituent cells occurred during adhesion. MSCs-GFP aggregate localized in a net of small blood vessels was shown in image (L). (A, D, G), fluorescence. (B, E, H), phase contrast. (C, F, I), merge. (J, K), fluorescence microscopy. (L), merge. (A-C, G-I, J, L), ×100; (K), ×200; (D-F), $\times 400$.
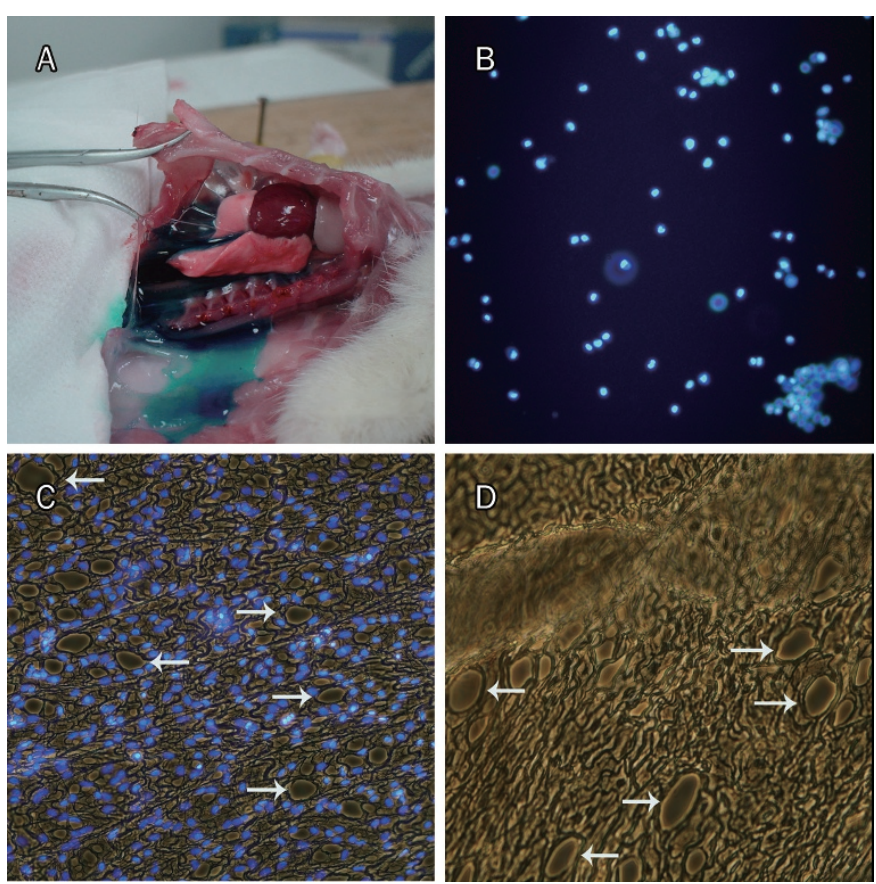

Figure 7. Channels exist for MSCs and fluid to traverse between the right and left pleural cavities. Only a few seconds after methylene blue injection into the right side pleural cavity, blue fluid was found flowing into the left thorax $(A)$, indicating that there were quick fluid channels that permitted fluid to pass through the mediastinum. Likewise, DAPI-labeled MSCs suspension was also injected into the right side pleural cavity, fluorescence cells were easily visualized in the fluid flowing into left thorax under fluorescence microscopy (B). Both images of DAPI-stained (C) and normal mediastinal pleura (D) demonstrated that there were many oval stomas of different sizes in the cell monolayer part of mediastinal pleura (white arrows), indicating that these may be the unidentified anatomical channels by which fluid and cells traverse between the two pleural cavities freely. (B-D), $\times 100$. 

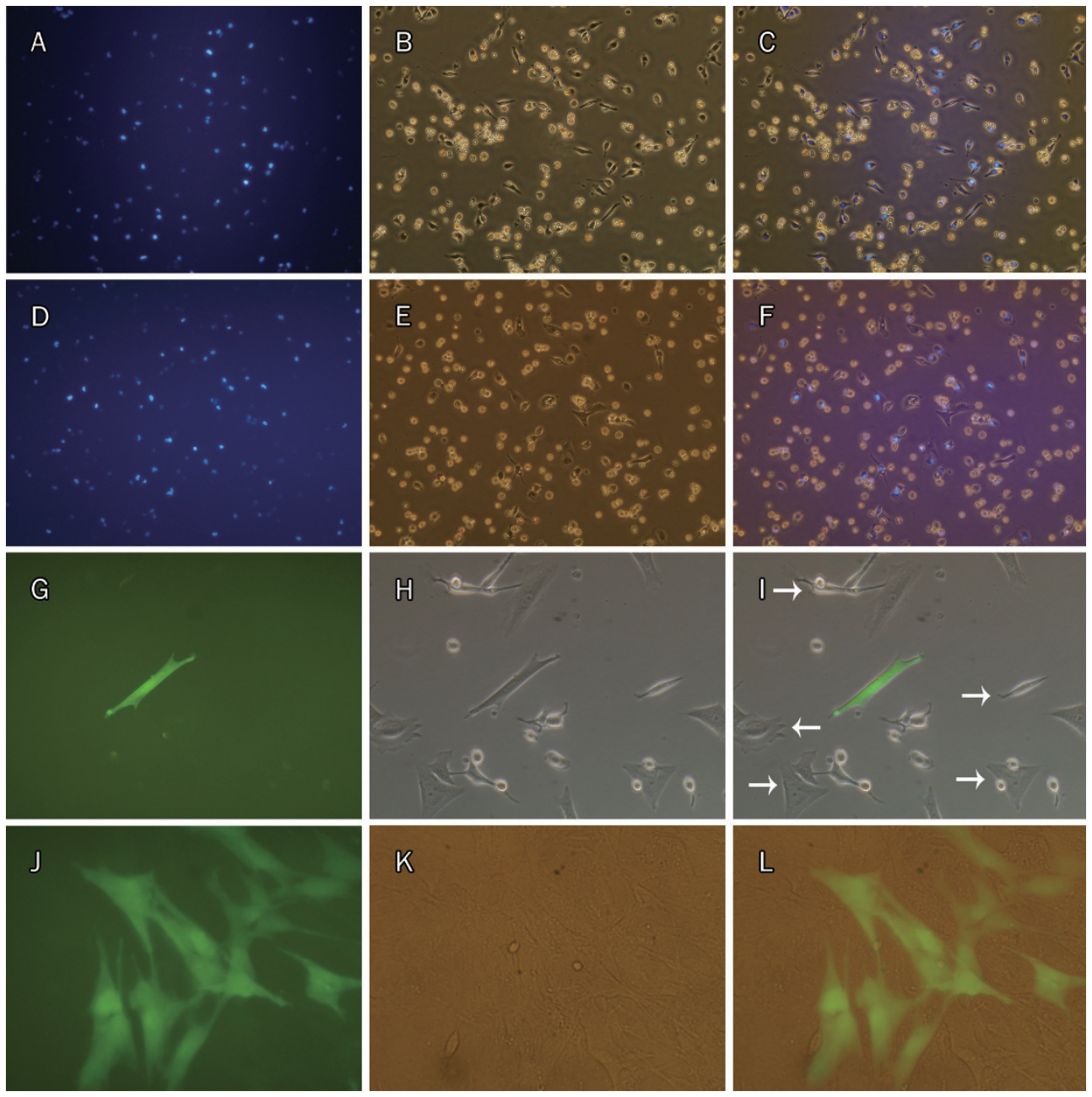

Figure 8. MSCs-DAPI and MSCsGFP recovered from the pleural cavity and cultured in vitro again. MSCsDAPI recovered were cultured in vitro for $1 \mathrm{~d}$ after being inoculated for 14 $d(A-C)$ and for $30 d(D-F)$. MSCsGFP recovered were cultured in vitro for $3 \mathrm{~d}(\mathrm{G}-\mathrm{H})$ and $17 \mathrm{~d}(\mathrm{~J}-\mathrm{L}, 7 \mathrm{~d}$ after passaged once) after being inoculated for $1 \mathrm{~d}$. Both recovered MSCs-DAPI and MSCs-GFP have the capability to adhere. However, only MSCs-GFP can proliferate. Beside MSCs-GFP, native cells from the pleural cavity also have property to adhere and proliferate (white arrows in I). (A, D, G, $J)$, fluorescence microscopy. (B, E, H, K), phase contrast. (C, F, I, L), merge. (A-F), $\times 200 ;(G-L), \times 400$.
MSCs-DAPI from all time points died quickly, while some of the native cells with the capacity to adhere proliferated and gradually became the predominating cells, the indentity of which still need to be determined. Compared with MSCsDAPI, inoculated MSCs-GFP could also be recovered from the pleural cavity on $\mathrm{d} 1$. When cultured and passaged in vitro again, both colonies of MSCs-GFP and those of native cells formed at the same time, and the total cell number increased, indicating that MSCs-GFP had capacity not only to adhere but also to proliferate (Figure 8G-8L).

\section{Discussion}

In this study, we have established and characterized a novel intrapleural method to deliver MSCs in vivo for the future treatment of pleural diseases. Unlike classical intravenous or intratracheal delivery methods ${ }^{[5-9]}$, intrapleurally delivered MSCs were found to be distributed only to the pleurae and the pleural cavity, providing a promising cell-based therapeutic strategy for primary and secondary pleural diseases.

As recommended in the workshop report written by Weiss et al that published in 2008 ${ }^{[12]}$, development of methods for culture systems that mimic in vivo niches was one of the strategies for a better understanding of the repair mechanisms of stem cells and their microenvironmental niche. The air-liquid interface, three-dimensional scaffolding/artificial matrices, the application of multiple cell types and mechanical forces (stretch, strain, etc) are four important factors suggested in this report for in vitro MSCs culture systems. Inspired by this, we initially thought that the pleural cavity might be an ideal target for MSC inoculation. As far as we are concerned, the pleural cavity is a tightly closed three-dimensional and potential compartment with negative pressure and multiple cell types and is like a "complex culture flask" in vivo; it satisfies most of the criteria. Most importantly, if inoculated MSCs can survive, proliferate, and be recovered viably by trypsinization like they can in an in vitro culture system, it would be an excellent model for MSC culture in vivo. At the same time, we hypothesized that this novel delivery method might have the potential to provide a new cell-based therapeutic strategy for pleural diseases.

Different pleural diseases may preferentially involve different parts of the pleura. For example, pleural mesothelioma has a tendency to affect the parietal pleura; in contrast, recurrent pneumothorax has a tendency to affect the visceral pleura. Therefore, we consider it necessary to ascertain if there is any difference in MSCs with regard to adherence to different parts 
of the pleurae. Results of this study showed that MSCs only adhered to the surfaces of the two pleural cavities (i.e. left and right), except for a small number of MSCs in several animals that were able to penetrate the cardiac pericardium and adhere to the surface of the epicardium in the pericardial cavity. No labeled MSCs were found in the lung parenchyma or in other tissues/organs distal to lung, such as the liver, the kidney, the spleen and the mesenterium. In addition to the adherence to the surfaces of the pleural cavities, a considerable number of MSCs were found in a suspended state in the two pleural cavities, indicating that a significant proportion of injected MSCs did not adhere to any surface. Although it is hard to ascertain the total number of suspended and adhered MSCs at the different time points, the number of adhered MSCs appeared to decrease over time. This likely reflects toxicity or another effect of DAPI rather than any inherent property of the MSCs or the pleural environment, as indicated by the growth curve analysis in the study. Further studies using Ki67 staining or some other marker of proliferation would be helpful to clarify this point. Unlike DAPI, a nuclear dye with intense fluorescence, GFP is expressed by the labeled cell itself, so its fluorescent signal is not attenuated by cell division and is more stable than that of DAPI. As it is expressed in the cytoplasm, GFP is better for showing the whole cell shape. Both DAPI- and GFPlabeling methods were used in this study to compare and validate the experimental results. MSCs-GFP were easily found in the mediastinal pleurae and translucent thymus as were MSCs-DAPI, but they were not found in other thick tissues like the surface of the diaphragm or the lungs. This could be explained by the fact that both the fluorescent signal of MSCsGFP and the contrast between them and their background due to autofluorescence were much weaker when compared with that of MSCs-DAPI. The fact that a large number of MSCsDAPI but not MSCs-GFP was found in pleural fixative fluid also suggests toxicity of DAPI. The formation of a "Halo" around MSCs-GFP or MSCs-DAPI aggregate on d 1 indicated that complex interactions had occurred between the MSCs and the pleural constituent cells during adhesion. This process may have included the production of adhesion molecules and the secretion of extracellular matrix. Furthermore, this phenomenon disappeared after $\mathrm{d} 1$, indicating that the process of adhesion might have been completed. Our results confirm the hypothesis that inoculated MSCs can survive and be limited to the pleural cavities. Because most of the cell adherence that occurred was confined to the visceral and the parietal pleura, it can reasonably be deduced that the administration of MSCs within the pleural cavity may have potential direct and indirect beneficial effects for the treatment of primary and secondary pleural diseases.

Most of the adhered MSCs were found on the mediastinal pleurae. This phenomenon indicated that the mediastinal pleura was the most favorable tissue for MSC adhesion. This may be due to the specific structure of the mediastinal pleura, a thin and relatively free membrane that separates the two pleural cavities. MSCs were prone to adhere near small blood vessels, implying that it might be a more convenient location for them to acquire nutrition from blood. Unlike cells cultured in vitro that grew as monolayers, MSCs that adhered in vivo were found to grow in a three-dimensional style, showing patterns of cell aggregates of different forms and scattered cells. We hypothesized that this may be mainly related to the tightly sealed and three-dimensional construction features of the pleural cavity or that it might result from interactions between MSCs and the pleural mesothelium cell microenvironment.

Interestingly, we found that MSCs could migrate into the left pleural cavity after inoculation into the right side. Subsequent experiments confirmed that there were indeed physical channels existing in the mediastinal pleura of rats, implying that the anatomy of rat mediastinal pleura is different from that of human beings. Although stomas on the costal pleura and the diaphragm have been identified and confirmed previously $^{[15,16]}$, there was previously no report on stomas on the mediastinal pleura, which is a thin membrane. Through light microscopy, we could observe stomas on the mediastinal pleura, as shown in Figure 7C-7D. Future studies using electron microscopy would be needed to further confirm this finding. The functions of these channels also need to be clarified in the future. This interesting result also indicates that injecting MSCs into one side of pleural cavity could have merit for the treatment of bilateral pleural diseases in an experimental rat model.

We also found that viable MSCs administered in vivo could be retrieved even $30 \mathrm{~d}$ post-inoculation. When cultured in vitro again, both MSCs-DAPI from all four time points and MSCs-GFP from d 1 have the capability to adhere. However, only MSCs-GFP proliferate, while MSCs-DAPI do not. This is not necessarily surprising since it was confirmed that the proliferation rate of MSCs decreased due to DAPI-labeling in this study. In addition, whether the recovered MSCs still retain characteristics exhibited prior to administration or whether these cells differentiated into host cells such as the mesothelium still needs to be characterized in future studies. Isolation of the inoculated MSCs from native cells trypsinized and recovered simultaneously should be done first, followed by flow cytometry and differentiation assays.

In summary, we have established and characterized a novel intrapleural method to deliver MSCs in vivo for the future treatment of pleural diseases. The distribution of intrapleurally delivered MSCs was limited to the pleurae and the pleural cavity, which may provide a novel way to further investigate the therapeutic roles of MSC-based therapy in pleural diseases.

\section{Acknowledgements}

The authors thank Xue-zhong QIN (Division of Regenerative Medicine, Department of Medicine, Loma Linda University School of Medicine, Loma Linda, CA, USA) for help in editing the manuscript and Bo SU from Shanghai Pulmonary Hospital for the FACS technical support. This study was supported by the National Natural Sciences Foundation of China (No 30971314 and 30800405), the Foundation of Shanghai Natural Science (No 08ZR1421000), the Postdoctoral Science Founda- 
tion (№ 20090460588), and the Shanghai Leading Talent Projects (No 036, 2010).

\section{Author contribution}

Zhao-hui QIN: Conception and design, collection and assembly of data, data analysis and interpretation, manuscript writing. Jie-ming QU: Conception and design, administrative support, manuscript writing, final approval of manuscript. Jin-fu XU: Manuscript writing. Jing ZHANG: Manuscript writing. Hanssa SUMMAH: Manuscript writing. He-Xi GE SAI-YIN, Chun-mei CHEN, and Long YU: Technical support.

\section{Supplementary information}

Supplementary figures are available at Acta Pharmacologica Sinica website of NPG.

\section{References}

1 Vorobiof DA, Mafafo K. Malignant pleural mesothelioma: medical treatment update. Clin Lung Cancer 2009; 10: 112-7.

2 Heffner JE, Klein JS. Recent advances in the diagnosis and management of malignant pleural effusions. Mayo Clin Proc 2008; 83: 23550.

3 Kass SM, Williams PM, Reamy BV. Pleurisy. Am Fam Physician 2007; 75: $1357-64$

4 Gupta A, Patel B, Almoosa K, McKelvy BJ. Recurrent spontaneous pneumothorax. Cleve Clin J Med 2010; 77: 345-7.

5 Xu J, Qu J, Cao L, Sai Y, Chen C, He L, et al. Mesenchymal stem cellbased angiopoietin-1 gene therapy for acute lung injury induced by lipopolysaccharide in mice. J Pathol 2008; 214: 472-81.

6 Gupta N, Su X, Popov B, Lee JW, Serikov V, Matthay MA. Intrapulmonary delivery of bone marrow-derived mesenchymal stem cells improves survival and attenuates endotoxin-induced acute lung injury in mice. J Immunol 2007; 179: 1855-63.

7 Xu J, Woods CR, Mora AL, Joodi R, Brigham KL, lyer S, et al. Prevention of endotoxin-induced systemic response by bone marrowderived mesenchymal stem cells in mice. Am J Physiol Lung Cell Mol Physiol 2007; 293: L131-41.

8 Zhao F, Zhang YF, Liu YG, Zhou JJ, Li ZK, Wu CG, et al. Therapeutic effects of bone marrow-derived mesenchymal stem cells engraftment on bleomycin-induced lung injury in rats. Transplant Proc 2008; 40: 1700-5.

9 Rojas M, Xu J, Woods CR, Mora AL, Spears W, Roman J, et al. Bone marrow-derived mesenchymal stem cells in repair of the injured lung. Am J Respir Cell Mol Biol 2005; 33: 145-52.

10 Horwitz EM, Prather WR. Cytokines as the major mechanism of mesenchymal stem cell clinical activity: expanding the spectrum of cell therapy. Isr Med Assoc J 2009; 11: 209-11.

11 Caplan Al. Why are MSCs therapeutic? New data: new insight. J Pathol 2009; 217: 318-24

12 Weiss DJ, Kolls JK, Ortiz LA, Panoskaltsis-Mortari A, Prockop DJ. Stem cells and cell therapies in lung biology and lung diseases. Proc Am Thorac Soc 2008; 5: 637-67.

13 Harting M, Jimenez F, Pati S, Baumgartner J, Cox C Jr. Immunophenotype characterization of rat mesenchymal stromal cells. Cytotherapy 2008; 10: 243-53.

14 Dominici M, Le Blanc K, Mueller I, Slaper-Cortenbach I, Marini F, Krause $\mathrm{D}$, et al. Minimal criteria for defining multipotent mesenchymal stromal cells. The International Society for Cellular Therapy position statement. Cytotherapy 2006; 8: 315-7.

15 Negrini D, Mukenge S, Del Fabbro M, Gonano C, Miserocchi G. Distribution of diaphragmatic lymphatic stomata. J Appl Physiol 1991; 70: 1544-9.

16 Wang NS. Anatomy of the pleura. Clin Chest Med 1998; 19: 229-40. 